\title{
Factores perinatales asociados a macrosomía fetal, en un hospital de Quito-Ecuador
}

\section{Factores perinatais associados à macrossomia fetal num hospital em Quito, Equador}

DOI: $10.46932 /$ sfjdv2n4-085

Received in: May 1st, 2021

Accepted in: Jun 30th, 2021

Paola Toapanta-Pinta

Médico Familiar-Máster en pediatría y neonatología.

Docente, carrera Obstetricia-Universidad Central del Ecuador.

Doctorado en Ciencias-Universidad de São Paulo.

Andrés Merizalde-Guerra

Médico- Servicio de Neonatología, Hospital Gineco Obstétrico Isidro Ayora.

Quito-Ecuador.

Paola Alarcón-Andrade

Médico-Servicio de Neonatología, Hospital Gineco Obstétrico Isidro Ayora.

Quito-Ecuador.

Cristhian Vasco-Toapanta

Estudiante-Escuela de ciencias de la Salud, Universidad San Francisco de Quito

Quito - Ecuador.

\section{Santiago Vasco-Morales}

Médico Pediatra-Servicio de Neonatología, Hospital Gineco Obstétrico Isidro Ayora.

Docente, Carrera de Obstetricia-Universidad Central del Ecuador.

Doctorado en Ciencias- Universidad de São Paulo.

Quito-Ecuador.

\section{RESUMEN}

OBJETIVOS: Determinar las condiciones perinatales relacionadas con macrosomía fetal, en nuestra población.

MÉTODOS: Estudio transversal de los registros del programa publicado por: El Centro Latinoamericano de Perinatología, Salud de la Mujer y Reproductiva, archivados en el Hospital Gineco-Obstétrico Isidro Ayora, desde enero del 2008 hasta diciembre del 2016. Para las variables numéricas se aplicó la prueba t. Las variables cualitativas se analizaron con la prueba Chi cuadrado. Para el análisis multivariado se utilizó modelos de regresión. En los resultados se incluye el Odds Ratio crudo y ajustado con el intervalo de confianza al 95\%. Los datos fueron analizados con el paquete estadístico R versión. 3.3.1 para Mac.

RESULTADOS: Se encontró 1171 (5.2\%) neonatos macrosómicos. Las variables asociadas a macrosomía fueron: diabetes, edad gestacional, edad materna, gestas previas, estado civil, sexo masculino, nacimiento vía cesárea, además existe asociación negativa con embarazo múltiple.

CONCLUSIONES: La macrosomía neonatal está relacionada con trastornos metabólicos en la madre, la mayor edad gestacional y materna, acompañamiento de la pareja durante el embarazo, la multiparidad, el producto masculino. El resto de los factores sociales, étnicos, las enfermedades crónicas y los trastornos propios del embarazo no mostraron asociación con macrosomía fetal. 
Palabras Clave: Macrosomía Fetal, Factores de Riesgo

\begin{abstract}
OBJECTIVES: To determine the perinatal conditions related to fetal macrosomia, in our population. METHODS: Transversal study corresponding to the program published by; Latin American Center of Perinatology, Women and Reproductive Health, archived at the Hospital Gineco Obstétrico Isidro Ayora, from January 2008 to December 2016. For the numerical variables, central tendency, dispersion measures, and the test $t$ were calculated. The relational analysis of the qualitative variables was performed with the Chi-square test, the multivariate analysis was performed by regression models. The crude and adjusted Odds Ratio is included in the results with the confidence interval value corresponding to $95 \%$. The database was analyzed using the statistical package $\mathrm{R}$ version. 3.3.1 for Mac.

RESULTS: 1171 (5.2\%) macrosomic neonates were found. The variables associated with macrosomia were diabetes, gestational age, maternal age, previous gestations, married woman, male sex, birth by cesarean section, and negative association with multiple pregnancies were also found.

CONCLUSIONS Neonatal macrosomia is related to metabolic disorders in the mother, gestational and maternal age, accompanying the husband during pregnancy, multiparity, the male product. The rest of the social, ethnic factors, chronic diseases, and disorders of pregnancy did not show an association with fetal macrosomia.
\end{abstract}

Keywords: Fetal Macrosomia, Risk Factors.

\title{
1 INTRODUCCIÓN
}

Se considera macrosómico, al neonato que al nacer tiene un peso de 4000 gr o más, este resultado obstétrico dado por un crecimiento intrauterino exagerado, que en la mayoría de los casos es multifactorial. La prevalencia de esta condición a nivel mundial se ha estimado en un $9 \%$, los estudios locales en el Ecuador indican porcentajes que van desde el 0,77\% hasta el 5\% (Åberg et al., 2016; Cannizzaro \& Paladino, 2011; Estrada-Restrepo et al., 2016; Gonzaga Aguilar, 2016; Guamán et al., s. f.; Koyanagi et al., 2013). Está establecido que el recién nacido macrosómico presenta mayor morbilidad por complicaciones tales como asfixia neonatal, lesiones de hombro, del plexo braquial, trastornos metabólicos, respiratorios, malformaciones congénitas, entre otros (Agudelo-Espitia et al., 2019). Además, a largo plazo y como consecuencia de la programación metabólica fetal en estos pacientes, existe mayor riesgo de desarrollar obesidad, en la etapa escolar, adolescencia y en la edad adulta, que han aumentado la incidencia de alteraciones musculo esqueléticas (Brian et al., 2021) además de las enfermedades crónicas no transmisibles en el adulto(Barker, 2007; Kehila et al., 2016; Luhete et al., 2016).

Los múltiples factores de riesgo asociados a macrosomía se han descrito en varios estudios, pero pueden presentar variaciones según las poblaciones estudiadas y estarían en relación con las condiciones fisiológicas, sociales y ambientales de la paciente embarazada, y cada uno puede tener diferentes grados de influencia. Entre los factores de riesgo comúnmente relacionados destacan, gestante con diabetes 
mellitus, desarrollo de diabetes gestacional, edad de la gestante mayor a los 35 años, obesidad, multiparidad, recién nacido (RN) posmaduro, sexo del RN(Agudelo-Espitia et al., 2019; Ballesté López \& Alonso Uría, 2004; Molina Hernández \& Monteagudo Ruiz, 2010).

El objetivo de este estudio fue determinar las condiciones perinatales que se asocian a macrosomía fetal en nuestro hospital.

\section{MÉTODO}

Se trata de un estudio transversal. Los datos fueron recolectados del SIP (Sistema Informático Perinatal), programa publicado por: El Centro Latinoamericano de Perinatología, Salud de la Mujer, archivados en el Hospital Gineco- Obstétrico "Isidro Ayora" de Quito (HGOIA), que es un hospital público, de referencia nacional, donde principalmente se atiende a usuarias de estrato económico medio bajo.

Luego de extraer los datos del Programa, se eliminó los nombres y número de registro tanto de la madre como del recién nacido y, se ingresó los datos en una hoja de cálculo. Como criterio de inclusión se consideró a los neonatos que tenían registrado en su base de datos el peso al nacer. Se excluyeron un total de 165 registros debido a ausencia de múltiples variables. Al disponerse de la base de datos completa, se decidió analizar la totalidad de los registros válidos, por lo que no fue necesario calcular el tamaño de la muestra.

Los datos de los RN se organizaron de acuerdo con su peso al nacer: Los $\mathrm{RN}$ que presentaron el peso al nacimiento entre 2500 hasta 3999 gramos, se asignaron al Grupo de Controles, y los RN que presentaron el peso al nacimiento mayor a 4000 gramos, se asignaron al Grupo de Casos.

Se estudió un total de 10.297 datos de neonatos vivos consecutivos. La variable endógena cuantitativa peso al nacer inicialmente fue analizada calculando la media poblacional y su respectiva desviación estándar (sd), para cada grupo de estudio de acuerdo con el sexo del RN, utilizando la prueba t.

Para el análisis de regresión, la variable endógena fue recodificada a binomial de acuerdo con la presencia o no de macrosomía, de la misma manera se recodificó a las variables exógenas:

a) Si existió o no el efecto: diabetes durante el embarazo o historia de diabetes, trastornos hipertensivos presentes durante el embarazo (hipertensión inducida por la gestación, eclampsia o preeclampsia), gestación múltiple, y vía del nacimiento por operación cesárea.

b) Las variables numéricas se recodificaron a dicotómicas nominales: número de consultas prenatales según las recomendaciones de la Organización Mundial de la Salud OMS (8 consultas y menos de 8 consultas), número de gestaciones anteriores (hasta 2 embarazos y más de 2 embarazos). La 
edad de la gestación se recodificó como: menor de 37 semanas o prematuro, a término si cumplía de 37 hasta $41^{+6}$ semanas y, postérmino si tenía más de 42 semanas de embarazo.

c) Variables nominales y politómicas: la condición de relación de pareja se codificó como soltera o unión estable. La edad de la madre se clasificó como adolescente, adulta y añosa de acuerdo con criterios universalmente establecidos. El grupo étnico con el que la madre de autoidentificó: indígena, blanca, afrodescendiente. El nivel académico alcanzado: primaria, secundaria, o estudios universitarios. La presencia y tipo de defectos congénitos: sin defectos, defectos mayores o menores.

Para entender la verdadera relación e influencia de las variables exógenas sobre la variable endógena, se desarrolló un análisis utilizando el modelo de regresión logística binaria (por la codificación de la variable endógena: si macrosomía, o peso adecuado), se realizó un análisis simple y posteriormente análisis múltiple. Para incluir las variables dentro del modelo final se procedió con los siguientes pasos:

1) Selección de las variables que en el modelo simple presentaron un menor valor del criterio de información, de Akaike.

2) Ajuste del modelo, mediante el test condicional de verosimilitudes, en este paso se incluyó las variables con menor valor de la devianza y valor $\mathrm{p}<0,05$.

3)Se comparó los dos modelos resultantes, para determinar cuál se ajustaba mejor a los datos, para este paso se realizó el análisis de Anova.

3) La validación del modelo final se ejecutó mediante el análisis de los residuos de la devianza, además del cálculo de las distancias de Cook.

Para el procesamiento de datos, se utilizó el paquete estadístico R Project for Statistical Computing 3.3.6, para Mac OS X.

Por tratarse de un estudio en fuente secundaria, se requirió la autorización del Comité de Docencia e Investigación del HGOIA. Los resultados iniciales de este trabajo están publicados en el libro de memorias del Congreso REDU basado en un reporte rápido presentado en un congreso en Ecuador (Vasco-Morales \& Toapanta-Pinta, 2017).

\section{RESULTADOS}

En el período estudiado, se encontró 1171 neonatos con peso al nacer $>4000$ gramos su distribución se presenta en la Tabla No 1. 
Tabla No.1 Neonatos con macrosomía, HGOIA 2008-2016

\begin{tabular}{lll}
\hline $\begin{array}{l}\text { Peso al nacer } \\
\text { en gramos }\end{array}$ & $\mathrm{n}$ & $\%$ \\
\hline $4000-4499$ & 1039 & 88.7 \\
$4499-5000$ & 122 & 10.4 \\
$>5000$ & 10 & 0.9 \\
\hline Total & 1171 & 100 \\
\hline
\end{tabular}

Fuente: Base de datos SIP-HGOIA.

Elaborado por: Los autores

Del total de neonatos macrosómicos ingresados a la sala de patología neonatal, el sexo masculino correspondió al 64\% n=749 la media de peso fue 4220.3 gr, sd 209.7 gr. Las mujeres $36 \%$ n=422, media de peso al nacer 4214, con un sd estándar de 208.1, valor p 0.5 .

Los resultados absolutos y relativos de las variables son presentados en las tablas 2 y 3 , tomando en cuenta que existió datos ausentes, el total de casos válidos estuvo entre 10085 y 10297, además se presenta el valor $\mathrm{p}$ de la prueba Chi cuadrado $\left(\mathrm{X}^{2}\right)$, para cada variable.

Tabla No. 2 Patologías crónicas y trastornos propios de la gestación.

\begin{tabular}{|c|c|c|c|c|c|c|}
\hline \multirow[b]{2}{*}{ Variables } & \multicolumn{2}{|c|}{ Macrosómico } & \multicolumn{2}{|c|}{ Peso adecuado } & \multirow{2}{*}{\multicolumn{2}{|c|}{$\begin{array}{lll}\text { total } & / & X^{2}\end{array}$}} \\
\hline & $\mathrm{n}$ & $\%$ & $\mathrm{n}$ & $\%$ & & \\
\hline Diabetes & & & & & 10297 & $\mathrm{p}<0.01$ \\
\hline No & 972 & 9.4 & 8999 & 87.4 & & \\
\hline $\mathrm{Si}$ & 51 & 0.5 & 275 & 2.7 & & \\
\hline Eclampsia & & & & & 10297 & $\mathrm{p}=0.2$ \\
\hline No & 1019 & 9.9 & 9226 & 89.6 & & \\
\hline $\mathrm{Si}$ & 4 & 0.03 & 48 & 0.5 & & \\
\hline Gestas previas & & & & & 10297 & $\mathrm{p}<0.01$ \\
\hline Mas de 2 & 351 & 3.4 & 1738 & 16.9 & & \\
\hline hasta 2 & 672 & 6.5 & 7536 & 73.2 & & \\
\hline
\end{tabular}

\begin{tabular}{lllllll}
$\begin{array}{l}\text { HTA inducida } \\
\text { por el embarazo }\end{array}$ & & & & & & \\
$\mathrm{No}$ & 957 & 9.3 & 8799 & 85.5 & & \\
$\mathrm{Si}$ & 66 & 0.6 & 475 & 4.6 & & \\
\hline HTA previa & & & & & 10297 & $\mathrm{p}=0.03$ \\
$\mathrm{No}$ & 1007 & 9.8 & 9162 & 89 & & $\mathrm{p}=0.16$ \\
$\mathrm{Si}$ & 16 & 0.2 & 112 & 1.1 & & \\
\hline Preeclampsia & & & & & 10297 & $\mathrm{p}=0.19$ \\
No & 888 & 8.6 & 8137 & 79 & & \\
$\mathrm{Si}$ & 135 & 1.3 & 1137 & 11 & & \\
\hline Violencia & & & & & 10297 & $\mathrm{p}=0.44$ \\
No & 1005 & 9.8 & 9105 & 88.4 & & \\
$\mathrm{Si}$ & 18 & 0.2 & 169 & 1.6 & & \\
\hline Cesárea & & & & & 10297 & $\mathrm{p}<0.01$ \\
No & 491 & 4.8 & 5741 & 53.1 & & \\
$\mathrm{Si}$ & 532 & 5.2 & 3803 & 36.9 & & \\
\hline
\end{tabular}

HTA: Hipertensión arterial

Fuente: Base de datos SIP-HGOIA.

Elaborado por: Los autores 
Recién nacido.

Tabla No. 3 Condiciones: fetales, económicas, sociales y ambientales

\begin{tabular}{lllllll}
\hline \multicolumn{2}{l}{ Macrosómico } & \multicolumn{2}{l}{ Peso adecuado } & \\
\hline Variables & $\mathrm{n}$ & $\%$ & $\mathrm{n}$ & $\%$ & total & $\mathrm{X}^{2}$ \\
\hline Defectos congénitos & & & & & & \\
Mayor & 33 & 0.3 & 682 & 6.9 & & $\mathrm{p}<0.01$ \\
Menor & 19 & 0.2 & 383 & 3.9 & & \\
No & 971 & 9.4 & 8209 & 89.2 & & \\
\hline Edad gestacional & & & & & 10295 & $\mathrm{p}<0.01$ \\
Prematuro & 20 & 0.1 & 928 & 9.1 & & \\
A término & 975 & 9.4 & 8259 & 80.2 & & \\
Postérmino & 28 & 0.2 & 85 & 0.8 & & $\mathrm{p}<0.01$ \\
\hline Sexo RN & & & & & \\
& & & & & \\
Femenino & 368 & 3.6 & 4074 & 39.6 & & \\
Masculino & 655 & 6.4 & 5199 & 50.5 & & \\
\hline
\end{tabular}

\section{Consultas prenatales adecuadas}

No

$\mathrm{Si}$

Edad materna

Adolescente

Adulta

Añosa

Embarazo múltiple

$\mathrm{No}$
$\mathrm{Si}$

Si

Soltera

Unión estable

\section{Estudios}

Ninguno

Primaria

Secundaria

Universidad

\section{Etnia}

Blanca

Indígena

Mestiza

Afrodescendiente
$605 \quad 6$

$402 \quad 4$

$129 \quad 1.3$

$714 \quad 6.9$

$\begin{array}{ll}180 & 1.7\end{array}$


En la tabla 4, se presenta únicamente las variables que en el análisis de regresión simple presentaron asociación con macrosomía, cada una con su valor OR crudo y ajustado al análisis múltiple, con su respectivo intervalo de confianza y el valor $\mathrm{p}$.

Tabla No. 4 Factores de riesgo asociados a macrosomía en el HGOIA

\begin{tabular}{|c|c|c|c|c|}
\hline & OR Crudo & $\mathrm{p}$ & OR Ajustado & $\mathrm{p}$ \\
\hline $\begin{array}{l}\text { Cesárea } \\
\text { Si/No }\end{array}$ & $1.55(1.36-1.77)$ & $<0.001$ & $1.74(1.51-2)$ & $<0.001$ \\
\hline $\begin{array}{l}\text { Diabetes } \\
\mathrm{Si} / \mathrm{No}\end{array}$ & $1.7(1.24-2.29)$ & $<0.001$ & $1.46(1.04-2)$ & 0.01 \\
\hline $\begin{array}{l}\text { Edad gestacional RN } \\
\text { Postérmino/ Prematuro }\end{array}$ & $16.81(8.93-32.55)$ & $<0.001$ & $21.04(10.91-41.85)$ & $<0.001$ \\
\hline $\begin{array}{l}\text { Edad gestacional } \mathbf{R N} \\
\text { A término/ prematuro }\end{array}$ & $6.02(3.83-10.18)$ & $<0.001$ & $7.45(4.66-12.86)$ & $<0.001$ \\
\hline $\begin{array}{l}\text { Edad materna } \\
\text { Adulta/ Adolescente }\end{array}$ & $2.68(2.22-3.27)$ & $<0.001$ & $2.1(1.71-2.59)$ & $<0.001$ \\
\hline $\begin{array}{l}\text { Edad materna } \\
\text { Añosa/Adolescente }\end{array}$ & $3.73(2.95-4.74)$ & $<0.001$ & $3.33(1.78-3.05)$ & $<0.001$ \\
\hline $\begin{array}{l}\text { Embarazo múltiple } \\
\mathrm{Si} / \mathrm{No}\end{array}$ & $0.55(0.3-0.93)$ & $<0.001$ & $0.79(0.2-0.75)$ & $<0.001$ \\
\hline $\begin{array}{l}\text { Estado civil } \\
\text { Unión estable /Soltera }\end{array}$ & $1.97(1.64-2.39)$ & $<0.001$ & $1.39(1.14-1.7)$ & $<0.001$ \\
\hline $\begin{array}{l}\text { Gestas previas } \\
\text { Hasta } 2 />2\end{array}$ & $0.44(0.38-0.5)$ & $<0.001$ & $0.56(0.48-0.61)$ & $<0.001$ \\
\hline $\begin{array}{l}\text { Sexo } \\
\text { Masculino/Femenino }\end{array}$ & $1.39(1.22-1.59)$ & $<0.001$ & $1.39(1.21-1.6)$ & $<0.001$ \\
\hline
\end{tabular}

RN: recién nacido.

Fuente: regresión logística de la base de datos SIP-HGOIA.

Elaborado por: Los autores

El modelo final fue validado a través del análisis de los residuos de la devianza, 532 presentaron un valor mayor a dos, correspondieron al 5\%, por lo que fue necesario estudiar su influencia mediante el cálculo de las distancias de Cook, ningún resultado se presentó mayor a 1. Por lo tanto, el modelo final, se consideró válido para determinar las asociaciones entre las variables.

\section{DISCUSIÓN}

En el grupo estudiado se encontró una prevalencia de macrosomía del 5.2\%, el elevado porcentaje se debe a que la macrosomía es causa de hospitalización neonatal para observación y estudio, tomando en cuenta además que el HGOIA es un hospital de referencia nacional. Si comparamos la cifra con reportes 
de estudios previos, hay países que presentan mayor prevalencia de macrosomía como Paraguay $(9,3 \%)$ y Cuba (7,2\%), mientras que la menor prevalencia en Ecuador (3,8\%) y Nicaragua $(2,9 \%)$ (̊berg et al., 2016; Koyanagi et al., 2013; Mainland \& Retnakaran, 2021).

La macrosomía está relacionada con el nacimiento por cesárea como procedimiento electivo debido al aumento de riesgo de desproporción céfalo-pélvica, distocia en el trabajo de parto, condiciones que con frecuencia llevan a complicaciones hipóxicas perinatales que pueden dar bajos puntajes de Apgar (Vasco et al., 2018) y sus consecuencias en el neurodesarrollo tal como ya se ha reportado en estudios realizado en esta población (Vasco-Morales et al., 2020) ; constituyéndose entonces como un procedimiento de terminación del embarazo más seguro tanto para la madre como para el feto frente a otras alternativas como el vacuum, con esta operación se espera disminuir el riesgo de trauma obstétrico, como lo confirma el trabajo de algunos autores (Åberg et al., 2016; Gonzaga Aguilar, 2016; Mainland \& Retnakaran, 2021; Usta et al., 2017).

Se encontró una débil asociación entre diabetes y macrosomía, la diabetes gestacional o previa, frecuentemente se relaciona con obesidad materna y/o intolerancia a la glucos; en conjunto son un grupo de alteraciones en el metabolismo de los carbohidratos, que predisponen a macrosomía fetal, y otras comorbilidades propias de esta condición incluido el bajo peso al nacer (Usta et al., 2017; Vasco et al., 2016). Estos resultados se podrían aclarar si se busca asociación entre macrosomía y síndrome del hijo de madre diabética, en donde además de las otras características de esta patología, tendremos que el paciente se encuentra sobre dos desviaciones estándar de la media en el índice de masa corporal de acuerdo con los estándares de la organización mundial de la salud (WHO, 2006).

Al igual que en los estudios de Estrada, Usta y Luhete, los embarazos postérminos tienen una mayor asociación con macrosomía, hallazgo similar se encontró con la edad materna; a mayor edad materna mayor asociación con macrosomía (Estrada-Restrepo et al., 2016; Kehila et al., 2016; Usta et al., 2017).

Como se aprecia en la tabla 4, mayor riesgo de macrosomía tienen los hijos de madres adultas y añosas en relación con las madres adolescentes, este resultado coincide con trabajos donde se evaluaron los factores de riesgo en gestantes adolescentes, incluidas las diabéticas (Bendezú et al., 2016; Rimbao Torres et al., 2007) donde no se encontró mayor riesgo macrosomía fetal en este grupo de madres. Este último dato contrasta con un estudio previo en esta misma población, donde no se encontró asociación con bajo peso al nacer en madres adolescentes (Vasco et al., 2016), datos que deben ser confirmados por estudios multicéntricos ya que al parecer el riesgo de presentar variaciones patológicas del peso al nacer es menor en este grupo de madres.

La preeclamsia, eclampsia, hipertensión inducida por el embarazo y otras enfermedades crónicas 
en la madre destacan por su fuerte asociación con bajo peso al nacer y restricción del crecimiento intrauterino, por lo que no se presentan como factores de riesgo para macrosomía fetal, tal como lo reportan los estudios locales y regionales(Estrada-Restrepo et al., 2016; Mainland \& Retnakaran, 2021; Vasco et al., 2016).

El embarazo múltiple se presenta como factor de protección para macrosomía, para poder interpretar adecuadamente este hallazgo, se debe considerar que la característica comúnmente asociada al embarazo múltiple es el peso bajo de acuerdo a los reportes locales y regionales(AJLAD et al., 2017; Toirac Lamarque et al., 2013). La condición civil asociada con macrosomía es la mujer en unión estable en comparación con la soltera, igual que lo hallado en Colombia (Estrada-Restrepo et al., 2016), se debe considerar que la mujer con pareja estable tiene mejor sustento económico, además de contar con el acompañamiento necesario durante este período de vulnerabilidad, por lo que el resultado obstétrico, comparado con la soltera sería un mayor peso al nacer. Se encontró que a medida que aumenta la paridad existe más asociación con macrosomía, está descrito que el mayor número de gestas previas y más aún si estos neonatos presentaron peso elevado al nacer, aumenta el riesgo de macrosomía fetal en los embarazos posteriores(Toirac Lamarque et al., 2013). A pesar de que no se evidenció una diferencia significativa en relación al peso y sexo de los neonatos macrosómicos, en este grupo se apreció una mayor prevalencia de macrosomía en el sexo masculino, dato que se repite, en los grupos poblacionales estudiados(AJLAD et al., 2017; Cannizzaro \& Paladino, 2011; Estrada-Restrepo et al., 2016; Luhete et al., 2016; Toirac Lamarque et al., 2013).

\section{CONCLUSIONES}

En este estudio se determinó que las condiciones perinatales asociadas con macrosomía fetal en el HGOIA son los trastornos metabólicos de la madre durante el embarazo como la diabetes, la mayor edad gestacional y posmadurez, madre adulta y sobre todo la añosa, las mujeres con pareja estable, la multiparidad, y en el neonato el factor de riesgo que destaca es el sexo masculino. En contraparte las otras características sociales, étnicas, el nivel de estudio, las patologías crónicas presentes durante el embarazo, así como los trastornos hipertensivos presentes durante la gestación, no presentaron asociación con macrosomía fetal en nuestra población.

Los autores declaran no tener ningún conflicto de interés.

El presente trabajo no contó con ninguna fuente de financiamiento. 


\section{REFERENCIAS BIBLIOGRÁFICAS}

Åberg, K., Norman, M., Pettersson, K. \& Ekéus, C. (2016). Vacuum extraction in fetal macrosomia and risk of neonatal complications: a population-based cohort study. Acta Obstetricia Et Gynecologica Scandinavica, 95(10), 1089-1096. https://doi.org/10.1111/aogs.12952

Agudelo-Espitia, V., Parra-Sosa, B. E. \& Restrepo-Mesa, S. L. (2019). Factors associated with fetal macrosomia. Revista de Saúde Pública, 53, 100. https://doi.org/10.11606/s1518-8787.2019053001269

AJLAD, C., Toro, M. S., Gutiérrez, C. \& Alarcón-Villaverde, J. (2017). Prevalence and associated factors of macrosomia in Peru, 2013. Revista peruana de medicina experimental y salud pública, 34(1), 36-42.

Ballesté López, I. \& Alonso Uría, R. M. (2004). Factores de riesgo del recién nacido macrosómico. Revista Cubana de Pediatría, 76(1), 0.

Barker, D. J. P. (2007). The origins of the developmental origins theory. Journal of Internal Medicine, 261(5), 412-417. https://doi.org/10.1111/j.1365-2796.2007.01809.x

Bendezú, G., Espinoza, D., Bendezú-Quispe, G., Torres-Román, J. S. \& Huamán-Gutiérrez, R. M. (2016). Características y riesgos de gestantes adolescentes . En Revista Peruana de Ginecología y Obstetricia (Vol. 62, pp. 13-18). scielo .

Brian, G. P., Flores, R. S., Aguiar, L. A. C., Zaleta, E. M., Carranco, R. G. H., Aidaly, A. M., Esther, A. R. M. \& Alfredo, M. V. J. (2021). Prevalencia de alteraciones músculoesqueléticas en niños escolares con obesidad en atención primaria. South Florida Journal of Development, 2(3), 4693-4705. https://doi.org/10.46932/sfjdv2n3-067

Cannizzaro, C. M. \& Paladino, M. A. (2011). Fisiología y fisiopatología de la adaptación neonatal. Anestesia Analgesia Reanimación, 24(2), 59-74. http://www.scielo.edu.uy/scielo.php?script=sci_abstract\&pid=S1688-

$12732011000200004 \& \operatorname{lng}=$ es\&nrm=iso\&tlng=es

Estrada-Restrepo, A., Restrepo-Mesa, S. L., Feria, N. D. C. C. \& Santander, F. M. (2016). Maternal factors associated with birth weight in term infants, Colombia, 2002-2011. Cadernos de Saúde Pública, 32(11). https://doi.org/10.1590/0102-311x00133215

Gonzaga Aguilar, D. A. (2016). Prevalencia de macrosomía en recién nacidos, con énfasis en madres que presentaron glicemias de 92 a 125mg/dl durante las primeras 24 semanas de edad gestacional y su asociación con factores de riesgo maternos, atendidas en el servicio de Gineco-Obstetric. PUCE.

Guamán, L. A. B., Macancela, M. E. I. \& Molina, M. A. M. S. (s. f.). CARACTERÍSTICAS DE LOS RECIÉN NACIDOS MACROSÓMICOS ATENDIDOS EN EL "HOSPITAL VICENTE CORRAL MOSCOSO” DURANTE EL PERIODO 2009-2014.

Kehila, M., Derouich, S., Touhami, O., Belghith, S., Abouda, H. S., Cheour, M. \& Chanoufi, M. B. (2016). Macrosomia, shoulder dystocia and elongation of the brachial plexus: what is the role of caesarean section? The Pan African Medical Journal, 25, 217. https://doi.org/10.11604/pamj.2016.25.217.10050

Koyanagi, A., Zhang, J., Dagvadorj, A., Hirayama, F., Shibuya, K., Souza, J. P. \& Gülmezoglu, A. M. (2013). Macrosomia in 23 developing countries: an analysis of a multicountry, facility-based, cross- 
sectional survey. Lancet (London, England), 381(9865), 476-483. https://doi.org/10.1016/S01406736(12)61605-5

Luhete, P. K., Mukuku, O., Kiopin, P. M., Tambwe, A. M. \& Kayamba, P. K. M. (2016). Fetal macrosomia in Lubumbashi: risk factors and maternal and perinatal prognosis. The Pan African Medical Journal, 23, 166. https://doi.org/10.11604/pamj.2016.23.166.7362

Mainland, R. \& Retnakaran, R. (2021). Maternal weight before and during pregnancy in women with gestational diabetes: one step forward, one step back «?. SciELO Brasil.

Molina Hernández, O. R. \& Monteagudo Ruiz, C. L. (2010). Caracterización perinatal del recién nacido macrosómico. Revista Cubana de Obstetricia y Ginecología, 36(3), 313-321.

Rimbao Torres, G., Cruz Hernández, J., Safora Enríquez, O., Rodríguez Izquierdo, A., Morales Chamizo, M. \& Velasco Boza, A. (2007). Comportamiento de la diabetes gestacional en el embarazo en la adolescencia . En Revista Cubana de Medicina General Integral (Vol. 23, p. 0). scielocu .

Toirac Lamarque, A. S., Pascual López, V., Martínez Jiménez, A. \& Area Suárez, R. I. (2013). Macrosomía fetal en madres no diabéticas. Caracterización mínima. Medisan, 17(10), 6052-6062.

Usta, A., Usta, C. S., Yildiz, A., Ozcaglayan, R., Dalkiran, E. Sen, Savkli, A. \& Taskiran, M. (2017). Frequency of fetal macrosomia and the associated risk factors in pregnancies without gestational diabetes mellitus. The Pan African Medical Journal, 26, 62. https://doi.org/10.11604/pamj.2017.26.62.11440

Vasco-Morales, S., Herrera-Tasiguano, A. E., Verdesoto-Jácome, C. A. \& Toapanta-Pinta, P. C. (2020). ESTUDIO PREDICTIVO DE LOS FACTORES DE RIESGO PERINATALES RELACIONADOS CON ALTERACIONES EN EL NEURODESARROLLO / PREDICTIVE STUDY OF PERINATAL RISK FACTORS RELATED TO NEURODEVELOPMENTAL DISORDERS. Brazilian Journal of Development, 6(9), 67931-67940. https://doi.org/10.34117/bjdv6n9-287

Vasco-Morales, S. \& Toapanta-Pinta, P. (2017). Factores de riesgo para macrosomía fetal. Hospital Gineco Obstétrico Isidro Ayora (HGOIA), Quito.

Vasco, S., Herrera, A., Acosta, M. \& Toapanta, P. (2018). Factores de riesgo para recién nacidos con Apgar bajo. Rev. Latin. Perinat., 21(3), 138-145. https://doi.org/10.5281/ZENODO.3772162

Vasco, S., Luzuriaga, S., Vélez, J., Acosta, M. \& Grandi, C. (2016). Factores de riesgo para bajo peso al nacer. REVISTA LATINOAMERICANA DE PERINATOLOGÍIA, 19(4), 236-241.

WHO, (Organization World Health). (2006). WHO child growth standards based on length/height, weight and age. Acta Paediatr Suppl, 450, 76-85. 